\title{
Confirmación de la expectativa vacación-estudio como factor principal del ritmo anual de incidencia y edad de menarquia
}

\author{
Carlos Y Valenzuela
}

\author{
Expectancy of vacation \\ as a determining factor \\ of the annual rhythm of menarche
}

Background: Our previous studies have shown that the monthly rhythm of menarche is associated to the expectancy of holidays or vacations. If this hypothesis is true, menarche episodes in Chile should cluster in December, the month prior to summer vacations. Aim: To study whether the expectancy of holidays or vacations is the main determinant of the annual rhythm of menarche. Material and methods: During 1990 and 1991, a questionnaire about the date of birth and date of menarche, was applied to girls living in Santiago (Chile) and Medellin (Colombia), aged 8 to 18.5 years. They were requested to register the year, month or day of menarche, only if they recalled it precisely. Results: Discarding clerical errors, data was obtained from 3,225 Chilean and 3,435 Colombian girls. Of these 73 and $63 \%$ of Chilean and Colombian girls recalled the day of menarche. Among Chilean girls an excess of menarche episodes was observed in December, January and February. The mean age per month of menarche reached its highest value of $155.6 \pm 15$ months in December and fell to $148 \pm 17$ months in January ( $p<0.001$ ). Among Colombian girls, mean age per month of menarche was also higher in December and lower in January $(152 \pm 15$ and $147 \pm 14$ months respectively, $\mathrm{p}<0.001$ ). However a peak of $152 \pm 15$ months was also observed in June. Conclusions: A similar distribution of menarche episodes was observed among Chilean and Colombian girls, with a peak in the months preceding holidays. The influence of the photoperiod is unlikely since Medellin is located in parallel 6 and there are little variations in daylight exposure in the different seasons. Therefore, our data supports the hypothesis that the prospect of holidays is a determinant of menarche (Rev Méd Chile 2006; 134: 606-12).

(Key words: Holidays; Menarche; Seasons)

Recibido el 1 de junio, 2005. Aceptado el 12 agosto, 2005.

Programa de Genética Humana, ICBM, Facultad de Medicina, Universidad de Chile. Santiago, Chile.

Correspondencia a: Carlos Y Valenzuela. Programa de Genética Humana, ICBM, Facultad de Medicina, Universidad de Chile. Independencia 1027, Casilla 70061, Independencia, Santiago, Chile. Fax (56-2) 7373158; Phone (56-2) 6786456-6786302.

E-mail: cvalenzu@med.uchile.cl 
$\mathrm{M}$ ás de 70 años de estudios no han encontrado el factor principal del ritmo de incidencia mensual de menarquia (RIMM) ${ }^{1-11}$, posiblemente porque los estudios son descriptivos, de asociación entre factores y ritmo de menarquia, o de ajuste de modelos matemáticos a los datos, pero no son hipotético-deductivos que sometan a prueba las situaciones o valores esperados de los factores supuestos causales de este ritmo. Después de nuestro trabajo sobre menarquia en Santiago (Chile) $)^{12}$, iniciamos un estudio hipotético-deductivo para dilucidar los factores causales del RIMM sea en su incidencia mensual o en el promedio mensual de edad de menarquia (EDM). Es evidente que cualquier factor responsable del RIMM también debe tener una fluctuación anual concordante. Desafortunadamente, los estudios agrupan los meses en estaciones u otras categorías o utilizan clases agrupadas de niñas según menarquia temprana o tardía, con lo que es imposible un análisis no sesgado 6,8-11, que lleva a una circularidad epistémica ${ }^{13}$. Refutamos, concluyentemente, la estacionalidad climática del fotoperíodo y de la temperatura ${ }^{14-16}$. Otros estudios contribuyeron a esta refutación ${ }^{7-9,11}$. Puesto que algunos trabajos, incluidos los nuestros, sugerían una relación entre el RIMM y el ritmo anual vacación-estudio 7,8,15, decidimos estudiar esta relación exhaustivamente en nuestros datos de India, Colombia, Hungría y Chile. Encontramos en estas 4 poblaciones que, de los 48 meses clasificados como de vacación o estudio, sólo 5 discreparon con la hipótesis que plantea que en los meses de vacación hay más menarquias que lo esperado por azar en el mes (cerca de 1/12) y en los de estudio se encuentran menos ${ }^{17}$. Esta discordancia o una más extrema, se da con una probabilidad menor que $7 \times 10^{-9}$, lo que indica una alta correlación entre el ritmo vacación-estudio y RIMM, que con tan baja probabilidad de errar alcanza casi condición de certeza biótica. Más aún, esos 5 meses discordantes eran mixtos vacación-estudio, por lo que es posible que la correlación fuese aún mayor con una clasificación vacación-estudio más estricta. En este trabajo también se volvió a refutar el factor climático como causa de RIMM. Si el mismo proceso ocurre en ciudades de India, Colombia, Hungría y Chile, podemos descartar los factores ambientales, nutricionales, étnicos, sociales o culturales como causales de RIMM, ya que ellos son muy diferentes en estos cuatro países. Debido a que los meses mixtos mostraban un comportamiento irregular y, que aparecía más bien la expectativa de vacación o estudio como el factor determinante, decidimos realizar un análisis, de acuerdo al día de menarquia, con las muestras de Chile y Colombia ${ }^{18}$. Encontramos que la expectativa de fiesta (vacación) o de no fiesta (estudio) se correlacionaban marcadamente con el RIMM. Las fiestas anuales relevantes para las niñas y sus cumpleaños presentaron alta incidencia de menarquia. El estudio presente pretende someter a prueba la hipótesis que el ritmo anual de menarquia descubierto por el análisis de su incidencia mensual, es producido por el ritmo anual de expectativas de [fiesta (vacación)] - [no fiesta (estudio)], pero ahora no desde la variable incidencia sino que desde la variable edad de menarquia (EDM). Es decir, el ritmo anual de expectativas causa un ritmo anual de las edades de menarquia (REDM). La influencia estacional sobre el REDM ha sido propuesta ${ }^{8,10}$. Estos estudios son importantes en antropología, psicología, psiquiatría y en gene$\mathrm{ral}$ para el conocimiento de las repercusiones somáticas de los estados mentales.

\section{Muestras y MÉTOdos}

Se aplicó un cuestionario a niñas escolares entre 8 y 19 años de edad en Santiago (Chile) y Medellín (Colombia). Las niñas registraron sus fechas de nacimiento con año, mes y día y de menarquia con año, mes y día, según recordaran. Se les solicitó que sólo registraran el año, mes o día, si los recordaban exactamente. Todos estos registros se realizaron con el consentimiento de niñas, padres, profesores y directores de colegios respectivos, según las normas éticas vigentes en ese momento. Creo importante mencionar que en nuestros estudios en el mundo, en India, Colombia y Chile, algunos establecimientos educacionales no aceptaron esta investigación en niñas menores de 11 años, por considerar que la encuesta les obligaba a introducir, según ellos, prematuramente temas de educación sexual sobre menstruación en esas edades. El método en su parte general y específico relacionado con la incidencia, la forma de evitar el sesgo de memoria ha sido extensivamente descrito en trabajos pre- 
vios $^{14-17}$. Descartando los errores clericales y considerando niñas con edades entre 8 y 18,5 años, obtuvimos 3.225 niñas chilenas y 3.435 niñas colombianas que recordaban al menos el año de menarquia. Cerca de $63 \%$ y de $73 \%$ de las niñas colombianas y chilenas, respectivamente, recordaban el día de menarquia. En este estudio incorporamos la edad de menarquia (EDM) como variable calculada en meses por la diferencia entre la fecha de menarquia y del nacimiento de 3 formas: I) EDM desde los meses y años de menarquia y nacimiento; II) EDM calculada en días para las niñas que recordaban el día de menarquia $\mathrm{y}$, luego dividida por el promedio de días que tiene un mes; III) EDM calculada agregando 15 días a la edad calculada en meses (para las niñas que sólo recordaban mes y año). Como todos los análisis resultaron iguales con los tres estimadores, sólo se describirán los resultados para EDM según III.

En Resultados y Discusión los meses serán abreviados con sus tres primeras letras en mayúscula.

Las niñas chilenas fueron encuestadas en 1990-1991 (1990-1), en Santiago (zona templada, latitud 33S, con 4 estaciones marcadas). En Chile, las fiestas cívico-religiosas más importantes ocurren en la última semana de diciembre y primero de enero (Navidad-Año Nuevo). Las fiestas nacionales más importantes son el 18-19 de septiembre (día patrio) y el 21 de mayo. Las vacaciones de verano ocurren entre mediados de diciembre y primeros días de marzo. Las vacaciones de invierno son 2 semanas en julio. En septiembre hay una semana de vacaciones en torno a los días patrios. El período de examen escolar es a fines de noviembre e inicios de diciembre. Las niñas colombianas fueron encuestadas en 1991 en Medellín (en la zona tórrida ecuatorial, latitud $6 \mathrm{~N}$, estacionalmente homogénea). Las principales fiestas cívico-religiosas ocurren el 8 de diciembre y entre Navidad y Año Nuevo (25 de diciembre al 1 de enero). El día patrio es el 20 de julio. Las vacaciones ocurren en: Semana Santa (variable entre marzo y abril); de medio-año entre el 20 de junio y 20 de julio; de verano fin de año entre el 20 de noviembre y el 31 de enero.

Una muestra chilena tomada en 1978 en Santiago ${ }^{12,14}$ y una brasileña de Riberão Preto (zona tropical latitud 21S, con muy poca variación estacional) ${ }^{11}$ servirán para poner a prueba la hipótesis [expectativa de fiesta-(no fiesta)] con EDM.

Puesto que la hipótesis estacional no ha sido puesta a prueba, en la variable edad, según nuestra metodología, la pondremos a prueba en este estudio, contrastándola con la hipótesis vacación-estudio. I) Si la causa de REDM es el ritmo anual estacional fotoperíodo-temperatura, entonces los promedios mensuales de EDM deben correlacionarse con este ritmo estacional: i) En Medellín y en Riberão Preto no debería presentarse un ritmo de EDM, ya que son climas tropicales con poca o ninguna variación estacional; ii) En Santiago, el ritmo de EDM debe ser evidente, los meses con similares fotoperíodo-temperatura deben tener similares EDM, los meses con distintos climas deben diferir en EDM. II) Si la causa de REDM es el ritmo expectativa vacación(fiesta)estudio(no fiesta) se espera un correlato entre ambos ritmos; en los meses de estudio deben tener su menarquia las niñas más tempraneras y el promedio mensual de EDM ir aumentando a medida que se acerca un período de vacación (el estudio retrasa la menarquia pero no la suprime); la expectativa de vacación, más el inicio de vacación, deben asociarse a un promedio de EDM más alto, hasta que todas las niñas retrasadas menstrúen por primera vez. En los estudios previos hubo un mínimo de incidencia de menarquia cercano al $5 \%$ en todos los países; como el promedio esperado es cercano a $1 / 12=8,3 \%$ se tiene que las niñas no sujetas (insensibles) a RIMM constituyen $5 / 8,3=60 \%$; el ritmo sería dado por el $40 \%$ de las niñas ${ }^{14-18}$. Otra interpretación puede ser que todas las niñas sean sensibles, con promedio de sensibilidad de 40\%. Conceptualmente utilizaremos la primera interpretación.

Estadística. Se utiliza ANOVA para las comparaciones de promedios mensuales con la corrección de Bonferroni para comparaciones múltiples. Las comparaciones pareadas de promedios de EDM (PEDM) o de incidencias se realizan con prueba t estándar o para proporciones, respectivamente ${ }^{19}$. El nivel de significación inicial es 0,05. Detalles se especifican en resultados. Los grados de libertad se sub-escriben. 


\section{RESUlTADOs}

La muestra chilena. La Tabla 1 presenta el número, porcentaje, PEDM con su desviación y error típicos, límites superior e inferior del intervalo de confianza de $95 \%$, para cada mes, encontrados en las niñas chilenas. Se observa un exceso de menarquias en DIC, ENE y FEB y una depresión en los otros meses. El análisis de la incidencia ha sido ya presentado ${ }^{14-18}$. El PEDM alcanza su máximo en NOV-DIC, cae en ENE y FEB y se mantiene en esas cifras hasta JUN (149 meses) y desde allí va aumentando hasta NOV-DIC, aunque AGO tiene un PEDM superior a JUL y SEP. El ANOVA para PEDM fue muy significativo $\left(\mathrm{F}_{11,3213}=9,99, \mathrm{P}<<10^{-6}\right)$; entre las 66 comparaciones de pares mensuales, 16 fueron significativas con la corrección de Bonferroni. La enorme diferencia entre NOV-DIC (PEDM=155,6 meses) y ENE-JUN (PEDM=149,0) de 7,1 meses fue muy significativa $\left(\mathrm{t}_{809}=6,49, \mathrm{P}<<10^{-6}\right)$. Tanto el cumpleaños como los días de fiestas que acumularon más menarquias que lo esperado (ver trabajo previo ${ }^{18}$ ), así como sus días circundantes no fueron distintos en PEDM respecto a los otros días del año.

La muestra colombiana. La Tabla 2 presenta los parámetros de la muestra de Medellín. El ANOVA para la distribución de PEDM fue altamente significativo $\left(\mathrm{F}_{11,3423}=4,8, \mathrm{P}<0,0001\right) ; 8$ entre 66 comparaciones de pares mensuales fueron significativas con la corrección de Bonferroni. La tendencia del PEDM fue similar a la de Santiago, con un máximo en NOV-DIC (151,9 meses) y mínimo en ENE-FEB (147,5 meses), prueba $t_{813}=4,38(P=0,00001)$. Hubo una diferencia notable con Santiago, en JUN se encontró un mayor PEDM que los encontrados en MAY y JUL, JUN y JUL, muestran un gran contraste en incidencia $\left(7,4 \%\right.$ vs $\left.16,3 \%, t_{810}=9,9, P<<10^{-9}\right)$ y en $P E D M$ $\left(152,0\right.$ vs 148,$\left.7 ; t_{810}=2,85, P=0,0045\right)$. Las altas incidencias de menarquias en el cumpleaños y fiestas $^{18}$ no se acompañaron de diferencias en el PEDM con respecto a los otros días del año, con excepción del 7 DIC, cuyo PEDM (161,8 meses, $\mathrm{N}=11, \mathrm{DT}=11,5)$ bordeó la significación $(\mathrm{P}=0,052)$ en relación al del 8 DIC (152,9 meses, N=28, $\mathrm{DT}=14,0)$.

Tabla 1. Incidencia y promedio de edad de menarquia mensuales. N iñas chilenas encuestadas en 1990-1991

\begin{tabular}{|lrrrrrrr|}
\hline & & & & & \multicolumn{3}{c|}{ Edad de menarquia en meses } \\
MES & $\mathrm{N}$ & $\%$ & PEDM & DT & ET & L & LS IC PEDM \\
\hline ENE & 415 & 12,87 & 148,46 & 16,79 & 0,8243 & 146,84 & 150,08 \\
FEB & 497 & 15,41 & 148,30 & 14,98 & 0,6717 & 146,98 & 149,62 \\
MAR & 238 & 7,38 & 149,12 & 14,46 & 0,9372 & 147,27 & 149,62 \\
ABR & 141 & 4,37 & 150,45 & 14,74 & 1,2411 & 148,00 & 152,91 \\
MAY & 142 & 4,40 & 150,77 & 14,49 & 1,2161 & 148,36 & 153,17 \\
JUN & 184 & 5,70 & 149,71 & 14,94 & 1,1015 & 147,53 & 151,88 \\
JUL & 250 & 7,75 & 151,32 & 13,87 & 0,8774 & 149,59 & 153,05 \\
AGO & 221 & 6,85 & 153,58 & 14,51 & 0,9760 & 151,66 & 155,50 \\
SEP & 283 & 8,78 & 151,61 & 14,78 & 0,8788 & 149,88 & 153,34 \\
OCT & 234 & 7,26 & 153,80 & 13,74 & 0,8981 & 152,03 & 155,57 \\
NOV & 224 & 6,95 & 155,75 & 13,20 & 0,8819 & 154,01 & 157,48 \\
DIC & 396 & 12,28 & 155,56 & 14,16 & 0,7117 & 154,16 & 156,96 \\
Total & 3.225 & 100,00 & 151,36 & 14,95 & 0,2632 & 150,84 & 151,87 \\
\hline
\end{tabular}

IC= intervalo de confianza; $\mathrm{N}=$ número; $\mathrm{PEDM}=$ promedio de edad de menarquia mensual; $\mathrm{DT}=$ desviación típica; ET=error típico; LI= límite inferior; $\mathrm{SL}=$ límite superior. 
Tabla 2. Incidencia y promedio de edad de menarquia mensuales. $N$ iñas colombianas encuestadas en 1991

\begin{tabular}{|lrrrrrrr|}
\hline & & & & & \multicolumn{3}{c|}{ Edad de menarquia en meses } \\
MES & $\mathrm{N}$ & $\%$ & PEDM & DT & ET & L & LS \\
\hline ENE & 334 & 9,72 & 147,90 & 13,62 & 0,7451 & 146,43 & 149,37 \\
FEB & 207 & 6,03 & 146,84 & 14,00 & 0,9732 & 144,93 & 148,76 \\
MAR & 208 & 6,06 & 148,14 & 13,92 & 0,9655 & 146,24 & 150,05 \\
ABR & 246 & 7,16 & 147,84 & 12,97 & 0,8267 & 146,21 & 149,46 \\
MAY & 229 & 6,67 & 148,77 & 14,26 & 0,9423 & 146,92 & 150,63 \\
JUN & 253 & 7,37 & 152,00 & 15,32 & 0,9633 & 150,10 & 153,89 \\
JUL & 559 & 16,27 & 148,70 & 15,28 & 0,6465 & 147,44 & 149,97 \\
AGO & 188 & 5,47 & 148,96 & 16,15 & 1,1779 & 146,63 & 151,28 \\
SEP & 177 & 5,15 & 149,86 & 13,90 & 1,0449 & 147,79 & 151,92 \\
OCT & 231 & 6,73 & 151,30 & 13,56 & 0,8923 & 149,54 & 153,06 \\
NOV & 322 & 9,37 & 151,43 & 15,20 & 0,8469 & 149,76 & 153,10 \\
DIC & 481 & 14,00 & 152,28 & 14,31 & 0,6524 & 151,00 & 153,56 \\
Total & 3.435 & 100,00 & 149,67 & 14,56 & 0,2484 & 149,18 & 150,16 \\
\hline
\end{tabular}

Nomenclatura como en la Tabla 1.

\section{DisCUSIÓN}

La concordancia entre lo esperado y observado para el promedio mensual de edad de menarquia (PEDM) tanto en Santiago como en Medellín, indica que hay un efecto real entre la expectativa vacación-estudio y el ritmo de edad de menarquia (REDM). Como esta relación se da también con la incidencia mensual de menarquia (RIMM), resulta más convincente que esta expectativa sea un factor mayor de ambos RIMM y REDM. Lo hallado en ambas ciudades refuta tanto la estacionalidad del proceso como el sesgo de memoria. No se explica que PEDM caiga entre DIC y ENE 7,1 meses en Santiago y 4,4 meses en Medellín por factores climáticos (son de la misma estación) o por sesgo de memoria. Al encontrar en Medellín tanto REDM como RIMM se refuta la estacionalidad, ya que es una ciudad ecuatorial casi sin estaciones. Se publican los parámetros directos para que el lector pueda encontrar otras refutaciones de la estacionalidad o del sesgo de memoria. Como NOV y DIC son meses de fines de estudio y de vacación y ENE es de vacación en ambos países, la explicación por la expectativa de vacación-estudio es inmediata. En NOV-DIC tuvieron su menarquia todas las niñas retrasadas por el régimen de estudio del año y en ENE se recupera el ritmo de menarquia sin retraso de todas las niñas por ser un mes de vacación. Desde MAR en Santiago y FEB en Medellín (con baja incidencia por ser de estudio) se empiezan a retrasar las niñas susceptibles. En Santiago hay un aumento de PEDM sostenido hasta NOV-DEC y, de acuerdo a lo esperado, en Medellín hay un brusco aumento en JUN (expectativa de vacación) para caer después en JUL y empezar a aumentar de nuevo hasta NOV-DIC.

Podemos someter a prueba esta hipótesis con muestras de Santiago colectados en 197812,14 y de Riberão Preto (Brasil) ${ }^{11}$ colectados en 1998 que se muestran en la Tabla 3. La muestra de Santiago es completamente concordante con la hipótesis tanto en incidencia como en PEDM. En Riberão Preto las vacaciones ocurren desde la última semana de DIC hasta fin de ENE y 2 semanas de JUL; FEB es un mes de estudio ${ }^{11}$. Con estos datos es evidente que en la muestra brasilera se da una concordan- 
Tabla 3. Incidencia y promedio de edad de menarquia mensuales. Niñas chilenas encuestadas en $1978{ }^{12,13}$ y brasileñas encuestadas en $1998^{11}$

\begin{tabular}{|c|c|c|c|c|c|c|c|}
\hline \multirow[b]{3}{*}{ MES } & \multicolumn{7}{|c|}{ Edad de menarquia en meses } \\
\hline & \multicolumn{4}{|c|}{ Muestra chilena 1978} & \multicolumn{3}{|c|}{ Muestra brasileña 1998} \\
\hline & $\mathrm{N}$ & $\%$ & PEDM & DT & $\mathrm{N}$ & $\%$ & PEDM \\
\hline ENE & 362 & 13,86 & 150,55 & 16,10 & 45 & 9,78 & 148,0 \\
\hline FEB & 344 & 13,17 & 149,59 & 16,75 & 35 & 7,61 & 144,3 \\
\hline MAR & 223 & 8,54 & 151,08 & 15,58 & 41 & 8,91 & 147,0 \\
\hline $\mathrm{ABR}$ & 160 & 6,13 & 150,08 & 16,88 & 28 & 6,09 & 151,0 \\
\hline MAY & 140 & 5,36 & 147,85 & 15,90 & 40 & 8,70 & 148,7 \\
\hline JUN & 187 & 7,16 & 149,87 & 14,55 & 42 & 9,13 & 150,2 \\
\hline JUL & 163 & 6,24 & 151,48 & 14,40 & 38 & 8,26 & 149,4 \\
\hline AGO & 180 & 6,89 & 152,87 & 15,18 & 39 & 8,48 & 150,0 \\
\hline SEP & 181 & 6,93 & 152,60 & 15,46 & 25 & 5,43 & 147,3 \\
\hline OCT & 186 & 7,12 & 152,97 & 15,67 & 28 & 6,09 & 149,1 \\
\hline NOV & 184 & 7,04 & 155,01 & 15,13 & 33 & 7,17 & 152,0 \\
\hline DIC & 302 & 11,56 & 157,68 & 13,54 & 66 & 14,35 & 152,7 \\
\hline Total & 2.612 & 100,00 & 151,92 & 15,69 & 460 & 100,00 & 149,4 \\
\hline
\end{tabular}

Nomenclatura como en la Tabla 1.

cia completa con la hipótesis de vacación-estudio. Entre NOV-DIC (PEDM=152,5 meses) y ENE-FEB (PEDM=146,4 meses) PEDM cayó 6,1 meses. DIC y ENE (14,4\% y 9,8\%) muestran un exceso y FEB $(7,6 \%)$ muestra una depresión de menarquias según lo esperado por la hipótesis de la expectativa de [fiesta (vacación)-estudio (no fiesta)]. En esta región tampoco se espera REDM ni RIMM por ser tropical, con lo que se refuta independientemente la estacionalidad del ritmo.

El enorme correlato entre la expectativa de vacación-estudio y variables independientes como

\section{REFERENCIAS}

1. VALSIK JA. The seasonal rhythm of menarche. A review. Hum Biol 1965; 37: 75-90.

2. BoJIEN K, BeNTZON WW. Seasonal variation in the occurrence of menarche. Dan Med Bull 1974; 21: 161-8.

3. BRundtLANd GH. Seasonal variations in menarche in Oslo. Ann Hum Biol 1982; 9: 35-43. incidencia y promedio de edad de menarquia mensuales indica que esta asociación procede de un fenómeno biótico real. Este correlato implica incidencias mensuales de menarquia tan dispares como 16\% y 5\% o diferencias de hasta 6 meses en PEDM en meses consecutivos. Parece ser que estas expectativas modifican enormemente los estados neuro-endocrinos en las niñas. El estudio apunta al hecho que en salud y enfermedad parece ser que las expectativas sobre el futuro de una persona pueden tener igual o más importancia que lo que le sucede en el presente. 
6. Albright DL, Voda AM, Smolensky MH, His BP, DECKER M. Seasonal characteristic of and age at menarche. Chronobiol Int 1990; 7: 251-8.

7. Wolanski N, Dickinson F, Siniarska A. Seasonal rhythm of menarche as a sensitive index of living conditions. Stud Hum Ecol 1993; 11: 17191.

8. GUERESI P. Monthly distribution of menarche in three Provinces of North Italy. Ann Hum Biol 1997; 24: 157-68.

9. Park SH, Shim YK, Kim HS, Eun BL Age and seasonal distribution of menarche in Korean girls. J Adolesc Health 1999; 25: 97.

10. Ayatolahi SMT, Dowlatabadi E, Ayatolahi SAR. Age at menarche in Iran. Ann Hum Biol 2002; 29: 355-62.

11. Tavares CH, Barbieri MA, Bettiol $\mathrm{H}$, Barbieri MR, De SouzA L. Monthly distribution of menarche among Schoolgirls from a municipality in Southeastern Brazil. Am J Hum Biol 2004; 16: 17-23.

12. Patri A, Valenzueia Cy, Morales I, Saavedra I, Figueroa L Edad de menarquia de niñas de Escuelas Fiscales del Área Norte de Santiago. Cuad Med Soc (Chile) 1980; 21: 12-20.

13. Valenzuela CY. Epistemic restrictions in population biology. Biol Res 1994; 27: 85-90.
14. Vaienzueia CY, NúÑEZ E, TAPIA C. Month at menarche: a re-evaluation of the seasonal hypothesis. Ann Hum Biol 1991; 18: 383-93.

15. Valenzuela Cy, Pineda N, Olarte G, Vásquez G. Ritmo anual de menarquia en Medellín, Colombia. Rev Méd Chile 1996; 124: 437-41.

16. Vaienzueia CY, Srikumari CR, Gopinath PM, Ghose $\mathrm{N}$, GajALAKSHMI P, CsOKNYAY J. New evidence of non seasonal factors in the menarche rhythm. Biol Res 1996; 29: 245-51.

17. Valenzuela CY, SRikumari CR, Csoknyay J, Pineda N. Ritmos anuales escolar (vacaciones-estudio) y de menarquia. Rev Méd Chile 1999; 127: 143-50.

18. ValenZuela CY. Día de menarquia, expectativa de fiesta y sesgo de memoria. Rev Méd Chile 2004; 132: 299-306.

19. Howew DC. Statistical Methods for Psychology, $5^{\text {th }}$ ed, Pacific Grove, CA: Duxbury, 2002.

Agradecimientos

In memoriam Dr. Alfredo Patrí M, que inició estos estudios. Estoy en deuda con CR Srikumari (India), J Csoknyay (Hungría), N Pineda (Colombia), E Núñez y C Tapia (Chile). Resultados presentados parcialmente en la XLVII Reunión Anual de la Sociedad de Biología de Chile, Pucón, Chile, 23-26 nov, 2004 [Biol Res 37(4) Suppl A, R96, 2004]. 